\title{
LEPTONIC DECAYS OF VECTOR MESONS: \\ THE BRANCHING RATIO OF THE ELECTRON-POSITRON DECAY MODE OF THE RHO MESON
}

\author{
J. G. Asbury,* U. Becker, William K. Bertram,*† P. Joos, M. Rohde, and A. J. S. Smith* \\ Deutsches Elektronen-Synchrotron, Hamburg, Germany \\ and
}

C. L. Jordan and Samuel C. C. Ting

Department of Physics, Columbia University, New York, New York (Received 25 July 1967)

At the Deutsches Elektronen-Synchrotron (DESY) 6.2-GeV electron synchrotron, experiments are in progress to investigate the leptonic decays of vector mesons. ${ }^{1}$ We report here a measurement of the branching ratio

$$
B=\Gamma\left(\rho^{0} \rightarrow e^{+} e^{-}\right) / \Gamma\left(\rho^{0} \rightarrow \pi^{+} \pi^{-}\right),
$$

from which the coupling constant $g_{\gamma \rho}$ may be obtained directly. To obtain (1), the rates of the two reactions

and

$$
\begin{aligned}
\gamma+\mathrm{C} \rightarrow \mathrm{C}+ & \rho^{0} \\
& \mathrm{~L} e^{+}+e^{-}
\end{aligned}
$$

$$
\begin{aligned}
\gamma+\mathrm{C} \rightarrow \mathrm{C}+\rho^{0} & \\
& \left\llcorner\pi^{+}+\pi^{-}\right.
\end{aligned}
$$

were measured under the same kinematical conditions and with the same apparatus, a symmetric double-arm magnetic spectrometer which has been described previously. ${ }^{2}$ The measurement of (3) is the subject of the preceding Letter, ${ }^{3}$ which is referred to as I in what follows.

To first order, both the Bethe-Heitler (BH) and Compton diagrams contribute to the reaction

$$
\gamma+\mathrm{C} \rightarrow \mathrm{C}+e^{+}+e^{-}
$$

For the case that the lepton pair $e^{+} e^{-}$has an invariant mass $M<600 \mathrm{MeV} / c^{2}$, the Compton term has been estimated ${ }^{4}$ and measured ${ }^{5}$ to contribute less than a few percent to (4) for pair production angles $\theta \leqslant 7^{\circ}$. Therefore, for $M .<600 \mathrm{MeV} / c^{2}$ Reaction (4) can be used as a sensitive test of quantum electrodynamics at small distances. ${ }^{2}$ As first suggested by Berman and Drell, ${ }^{1}$ however, the Compton contribution will be greatly enhanced if $M$ is equal to the mass of a vector meson $(\rho, \omega$, or $\varphi$ ); hence (4) can also be used to study the leptonic decays of vector mesons.

For a measurement of the branching ratio (1) to be meaningful, three major error sources must be reduced or avoided: (a) $\mathrm{BH}$ background must be kept small, as its subtraction contributes to (1) a statistical uncertainty as well as an uncertainty caused by lack of precise knowledge of inelastic carbon form factors. At symmetry, for a given pair invariant mass $M=2 p \theta$ ( $p$ is the momentum of the electron and positron, $\theta$ the angle of each with respect to the $\gamma$ beam), the $\mathrm{BH}$ counting rate varies roughly as $\theta^{-6}$, whereas the $\rho^{0} \rightarrow e^{+} e^{-}$pair rate varies as $\theta^{-3}$.

Therefore,

$$
\left(\rho \rightarrow e^{+} e^{-}\right) / \mathrm{BH} \approx \theta^{3}
$$

and the $\mathrm{BH}$ background can be reduced by observing $\rho^{0} \rightarrow e^{+} e^{-}$at large angles.

(b) The process $\omega \rightarrow e^{+} e^{-}$contributes to the measured $e^{+} e^{-}$rate at the $\omega$ mass. However, by using a spectrometer with a mass resolution comparable with the $\omega$ natural width, one would see any sizable contribution from this process as an enhancement in the $e^{+} e^{-}$invariant-mass spectrum. Similarly, a large interference effect between $\rho^{0}$ and $\omega$ could be detected, as it would cause a distortion in the shape of the $e^{+} e^{-}$mass distribution. However, there are arguments that the rate of $\rho^{0} \rightarrow e^{+} e^{-} \gg$ rate of $\omega \rightarrow e^{+} e^{-}$: Bubble-chamber data show for $\mathrm{H}_{2}$ that $\omega$ photoproduction $\simeq 10 \%$ of $\rho^{0}$ production. If $\rho^{0}$ and $\omega$ are produced by similar mechanisms, ${ }^{6}$ this holds for carbon as well. Then the $\mathrm{SU}(6)$ prediction ${ }^{7}$ of the ratio

$$
\gamma_{\rho}^{-2}: \gamma_{\omega^{-2}}: \gamma_{\varphi}^{-2}=9: 1: 2
$$

gives the result that $\omega \rightarrow e^{+} e^{-}$is only $10 \%$ of $\rho^{0} \rightarrow e^{+} e^{-}$.

(c) To obtain an accurate value of the branching ratio (1) both the $\rho^{0}$ production cross section and the $\rho^{0}$ polarization must be known over the kinematical region for which $e^{+} e^{-}$pairs are accepted. For this experiment, systematic errors caused by the $\rho^{0}$ cross-section measurement were reduced by measuring both (2) and (3) with the same apparatus. Also, the 
$\rho^{0}$ polarization was determined indirectly as implied in I, the conclusion being that $\rho^{0}$ production at forward angles proceeds via diffraction dissociation, the $\rho^{0}$, therefore, being completely transversely polarized. The angular distribution $W\left(\theta^{*}\right)$ in the $\rho^{0}$ rest system is then determined: For pion pairs,

$$
W_{\pi \pi}\left(\theta^{*}\right)=(3 / 8 \pi) \sin ^{2} \theta^{*},
$$

and for the electron pairs,

$$
W_{e e^{\left(\theta^{*}\right)}=(3 / 16 \pi)\left(1+\cos ^{2} \theta *\right)}
$$

( $\theta^{*}$ is the angle in the $\rho^{0}$ rest system between the decay products and the incident $\gamma$-ray direction).

On the basis of considerations (a)-(c) above, the experimental arrangement was chosen to minimize systematic errors as follows: First, the largest central pair opening angle consistent with a reasonable counting rate was chosen, namely $30^{\circ}$. The uncertainty in inelastic form factors then introduces an error $\approx 5 \%$ in the BH subtraction. Second, hodoscopes (described in Ref. 2) giving a mass resolution of $\pm 15 \mathrm{MeV} / c^{2}$ were used to enable $\omega \rightarrow e^{+} e^{-}$contributions to be observed. Finally, as described in $I$, errors in the $\rho^{0}$ production cross section due to the presence of nonresonant $\pi+\pi$ - background were reduced to $\pm 10 \%$ by the careful study of Reaction (3) for different target elements, momentum transfers, and incident peak bremsstrahlung energies $k_{\text {max }}$. (The bremsstrahlung beam is described in Ref. 2.)

The $e^{+} e^{-}$pair rate at $\theta_{0}=15^{\circ}$ and $p_{0}=1.400$ $\mathrm{GeV} / c$ (central pair mass $M_{0} \approx 750 \mathrm{MeV} / c^{2}$ ) was much larger than that predicted by quantum electrodynamics (QED). To show that this apparent deviation is caused by an enhancement in the Compton diagram and not by a fundamental QED breakdown (modification of Feynman propagator or vertex), a run was taken at $\theta_{0}$ $=9.5^{\circ}, p_{0}=2.25 \mathrm{GeV} / c\left(M_{0}\right.$ also $\left.\approx 750 \mathrm{MeV} / c^{2}\right)$. As shown in Fig. 1, the ratio

$$
R=\frac{\text { Experimental yield }}{\text { Bethe-Heitler yield }}
$$

increases rapidly with angle, in a manner consistent with Eq. (5), demonstrating that the large pair rate for $M \approx 750 \mathrm{MeV} / c^{2}$ can be explained without invoking a fundamental breakdown of QED.

Also shown in Fig. 1 is the value of $R$ obtained,

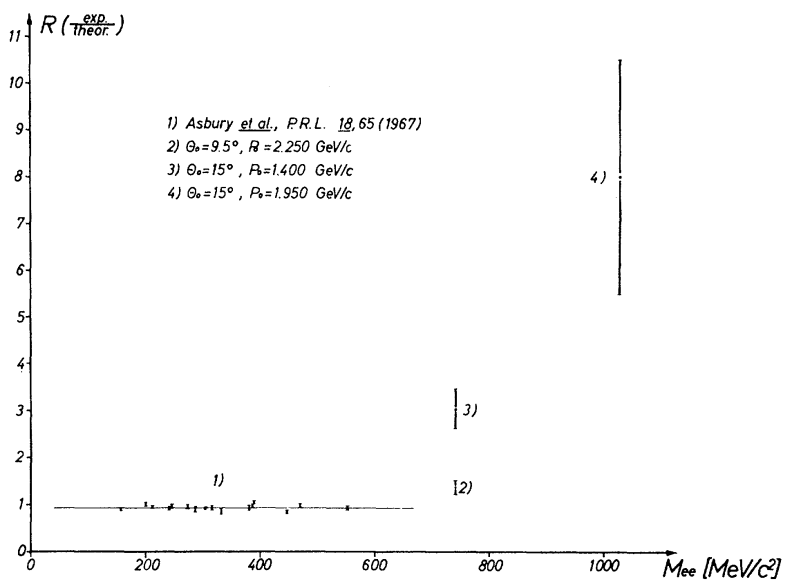

FIG. 1. The ratio $R=($ Experimental yield $) /($ Calculated $\mathrm{BH}$ yield) is shown as a function of the invariant mass of the $e^{+} e^{-}$pairs.

on the basis of ten events, for the spectrometer setting $\theta_{0}=15^{\circ}, p_{0}=1.95 \mathrm{GeV} / c\left(M_{0}=1020\right.$ $\left.\mathrm{MeV} / c^{2}\right)$. Here the observed $e^{+} e^{-}$pair rate is $\approx 7$ times that expected from $\mathrm{BH}$; this gives strong evidence that the decay $\varphi \rightarrow e^{+} e^{-}$has been observed.

The mass spectrum of $e^{+} e^{-}$events is shown in Fig. 2(a). The $\mathrm{BH}$ background has been subtracted, and all corrections have been applied. The spectrum is in agreement with the modi-

fied Breit-Wigner mass distribution ${ }^{8}$ of $\rho^{0} \rightarrow \pi^{+} \pi^{-}$ of I $\left(\Gamma_{\rho}=130 \pm 5 \mathrm{MeV}\right.$ and $\left.M_{\rho}=765 \mathrm{MeV} / c^{2}\right)$. The smoothness and approximate symmetry of the distribution indicates that the $\omega \rightarrow e^{+} e^{-}$ contribution is $<5 \%$.

The branching ratio (1) is now obtained by dividing the integrated yield of Fig. 2(a) by the yield of $\rho^{0}$ mesons produced in the same accepted region; this latter yield is shown in Fig. 2(b). Taking the polarization factors given in Eqs. (7) and (8) into account, we obtain the result ${ }^{9}$

$$
B=(6.5 \pm 1.4) \times 10^{-5} \text {. }
$$

As a direct application of our knowledge of the behavior of $\rho^{0}$ photoproduction cross sections on carbon [Eq. (2), preceding Letter] and $B$ determined in this experiment, the $\rho^{0}$ photoproduction amplitude (2) is determined experimentally to be of the form

$$
A_{\gamma \rho}=D(k) p e^{\frac{1}{2} a t} i e^{i \phi} /\left(m_{\rho}^{2}-m^{2}-i m_{\rho} \Gamma\right) \text {, }
$$

where $i e^{i \phi}$ is an arbitrary phase and where $D(k)$ normalizes the amplitude to the observed 
zero-degree $\rho^{0}$ photoproduction cross section. In the region of the $\rho^{0}$ mass the dominant virtual Comp-

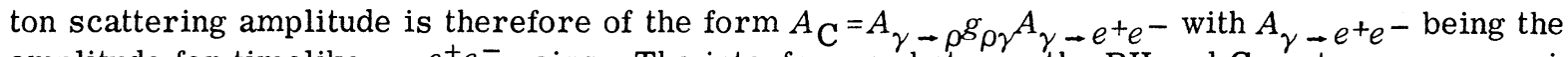
amplitude for timelike $\gamma \rightarrow e^{+} e^{-}$pairs. The interference between the $\mathrm{BH}$ and Compton processes is then described by the cross section

$$
\begin{aligned}
& d \sigma_{\text {int }} \equiv \frac{d \sigma}{d E_{+} d E E_{-} d \Omega_{+} d \Omega_{-}}=\frac{Z \alpha^{2}}{\pi^{2}} G_{E}\left(q^{2}\right) E_{+} E-\frac{e^{\frac{1}{2} a t} g}{t} \frac{\rho \gamma^{m} \rho}{m^{2}} G(k) \\
& \times\left[2 m^{2}\left(\frac{E-_{-}}{k \cdot p_{+}}-\frac{E_{+}}{k \cdot p_{-}}\right)+2\left(\frac{1}{k \cdot p_{+}}+\frac{1}{k \cdot p_{-}}\right)\left[\frac{1}{2} m^{2}\left(E_{+}-E_{-}\right)+k \cdot p_{+} E_{-}-k \cdot p_{-} E_{+}\right]\right. \\
& \left.-\frac{2}{M}\left(p_{+x} p_{-x}+p_{+y} p_{-y}\right)\left(\frac{Q \cdot p_{+}}{p_{-} \cdot k}-\frac{Q \cdot p_{-}}{p_{+} \cdot k}\right)\right] D(k) \operatorname{Re}\left[\frac{i e^{i \phi}}{m_{\rho}^{2}-m^{2}-i m_{\rho} \Gamma}\right],
\end{aligned}
$$

where $k=$ photon four-momentum; $G(k)=$ bremsstrahlung energy spectrum; $M=$ mass of target nucleus; $Q=k+P-D_{+}-D_{-}$(recoil four-momentum of nucleus); $P=$ initial four-momentum of nucleus; and $t=\left(k-p_{+}-p_{-}\right)^{2}$. The metric chosen is $g_{00}=1, g_{i i}=-1 \quad(i=1,2,3)$, and the $\gamma-\rho$ coupling constant $g_{\gamma \rho}$ is directly determined from the branching ratio $B$.

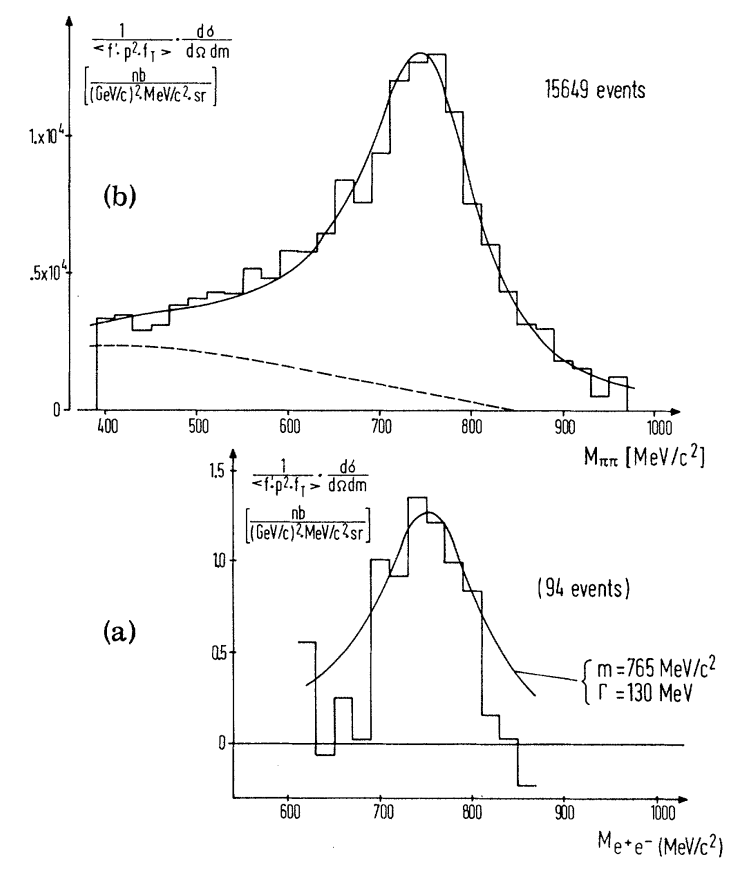

FIG. 2. (a) Pair yield from $\rho^{0} \rightarrow e^{+} e^{-}$as function of invariant mass. BH background has been subtracted, and the magnitude of the yield includes the effect of the complete polarization of the $\rho^{0}$ mesons. The peak bremsstrahlung energy $k_{\max }=6.0 \mathrm{GeV}$. (b) Pair yield from $\rho^{0} \rightarrow \pi^{+} \pi^{-}$as function of invariant mass. Again the effect of polarization of the $\rho^{0}$ mesons is included in the calculation of the yield. The momentum $p$ is $2.8 \mathrm{GeV} / c$, and $k_{\max }=6.0 \mathrm{GeV}$.
The interference cross section $d \sigma_{\text {int }}$ is asym metrical under interchange of four-momenta of electron $\left(p_{-}\right)$and positron $\left(p_{+}\right)$, and thus can be observed by taking the difference between two asymmetrical yields for spectrometer settings of opposite polarity. The resultant yield is then independent of any possible systematic asymmetry in the spectrometer as well as pure $\mathrm{BH}$ and Compton contributions.

Our measurements at $15^{\circ}, 1400 \mathrm{MeV} / c\left(m_{0}\right.$ $\left.=m_{\rho}\right)$, when compared with (9), give us $\phi=15$ $\pm 25^{\circ}$, consistent with the prediction of a purely absorptive $\left(\phi=0^{\circ}\right)$ Compton amplitude.

We thank Dr. J. Pumplin for pointing out the importance of only including transversely polarized $\rho$ 's in the derivation of Eq. (3). To him and to Dr. J. Fleischer we are grateful for performing careful checks of our original calculations. We thank Dr. M. Binkley for participation in the last part of the run. We are grateful for the enthusiastic support of Professor W. Jentschke and Professor P. Stähelin of DESY and Professor L. M. Lederman and Professor S. Devons of Columbia, which made this collaboration possible. We also thank Professor H. Joos, Professor G. Weber, Professor M. Ross, Professor S. D. Drell, and Dr. S. J. Brodsky for interesting discussions. We thank Dr. H. O. Wüster and Dr. D. Lublow for extending to us the DESY computing facilities, and Mr. H. Kumpfert and the synchrotron staff for their skillful operation of the machine.

*Volkswagen Foundation Fellow at DESY.

$\uparrow$ Present address: Physics Department, University 
of Illinois, Urbana, Illinois.

\$A DESY-Columbia collaboration, this work was supported in part by the U. S. Atomic Energy Commission.

${ }^{1}$ For theoretical papers on leptonic decay of vector mesons, see M. Gell-Mann, D. Sharp, and W. G. Wagner, Phys. Rev. Letters 8, 261 (1962); S. L. Glashow, Phys. Rev. Letters 7 , $46 \overline{9}$ (1961); M. Gell-Mann and F. Zachariasen, Phȳs. Rev. 124, 953 (1961); Y. Nambu and J. J. Sakurai, Phys. Rev. Letters 8, 79 (1962); S. M. Berman and S. D. Drell, Phys. Rev. 133, B791 (1964); N. M. Kroll, T. D. Lee, and B. Zumino, to be published; R. van Royen and V. F. Weisskopf, to be published; H. Joos, Phys. Letters 24B, 103 (1967); K. Kajantie and J. S. Trefil, Phys. Letters 24B, 106 (1967).

${ }^{2}$ J. G. Asbury, U. Becker, William K. Bertram, P. Joos, M. Rohde, A. J. S. Smith, S. Friedlander, C. Jordan, and Samuel C. C. Ting, Phys. Rev. Letters 18,65 (1967).

${ }^{3}$ J. G. Asbury, U. Becker, William K. Bertram, P. Joos, M. Rohde, A. J. S. Smith, C. L. Jordan, and Samuel C. C. Ting, preceding Letter [Phys. Rev. Letters 19,865 (1967)].

${ }^{4}$ A. Krass, Phys. Rev. 138, B1268 (1965); S. D. Drell, in Proceedings of the International Symposium on Electron and Photon Interactions at High Energies, Hamburg, 1965 (Springer-Verlag, Berlin, Germany, 1966), Vol I, p. 71.

${ }^{5}$ J. G. Asbury, U. Becker, Willíam K. Bertram, P. Joos, M. Rohde, A. J. S. Smith, C. L. Jordan, and Samuel C. C. Ting, to be published.

${ }^{6}$ Cambridge Bubble Chamber Group, in Proceedings of the International Symposium on Electron and Photon Interactions at High Energies, Hamburg, 1965 (Springer-Verlag, Berlin, Germany, 1966), Vol. II, p. 1; Phys. Rev. 146, 994 (1966); Phys. Rev. Letters 13, 640 (1964). German Bubble Chamber Collaboration, in Proceedings of the International Symposium on Electron and Photon Interactions at High Energies, Hamburg, 1965 (Springer-Verlag, Berlin, Germany, 1966), Vol. II, p. 36; Nuovo Cimento 41A, 270 (1966); and to be published; DESY Report No. 66/32 (unpublished).

${ }^{7}$ C. A. Levinson, H. J. Lipkin, and S. Meshkov, Phys. Letters $\underline{7}, 81$ (1963).

${ }^{8}$ J. D. Jackson, Nuovo Cimento 34, 1644 (1964).

${ }^{9}$ For up-to-date comparison of this work with other related results see Samuel C. C. Ting in Report to the 1967 International Conference on Photons and Electrons, Stanford University, September, 1967 (unpublished).

\title{
TEST FOR TIME-REVERSAL INVARIANCE OF THE ELECTROMAGNETIC INTERACTION USING THE MÖSSBAUER EFFECT IN $\mathrm{Ru}^{99} \dagger$
}

\author{
O. C. Kistner \\ Brookhaven National Laboratory, Upton, New York \\ (Received 1 August 1967)
}

The apparent violation of $C P$ invariance in the $2 \pi$ decay mode ${ }^{1}$ of the $K_{2}{ }^{0}$ has led Bernstein, Feinberg, and Lee ${ }^{2}$ to bring attention to the fact that there is no substantial experimental evidence that the electromagnetic interaction of the strongly interacting particles is invariant under $C$ and $T$. The se authors show that strong violation of $T$ invariance of the electromagnetic interaction may reveal itself, through higher order effects, as a $T$ noninvariant impurity of 0.1 to $1 \%$ in the amplitude of the nuclear gamma-decay matrix element. The invariance under $T$ requires that the relative phase angle $\psi$ between the individual multipole amplitudes of a mixed gamma transition be either 0 or $\pi$. A finite value of $\sin \varphi$ would be equal to the fraction of $T$-noninvariant amplitude present. The most sensitive experiments previously reported have employed $\beta-\gamma_{1}-\gamma_{2}$ triple coincidence measurements ${ }^{3}$ and $\gamma_{1}-\gamma_{2}$ coincidence measurements following polarized-neutron capture, ${ }^{4}$ and quote an upper limit for $\sin \varphi$ of the order of sever- al percent. The present experiment employs the Mössbauer effect on the 90-keV $M 1-E 2$ mixed transition ${ }^{5}$ in $\mathrm{Ru}^{99}$ and is an order of magnitude more sensitive, thereby bringing the measurement well within the region of interest.

Jacobsohn and Henley ${ }^{6}$ have tabulated the various possible quantities which are proportional to $\sin \varphi$. Of these, the term of the form $(\vec{k} \cdot \vec{j} \times \vec{\epsilon})(\vec{k} \cdot \vec{j})(\vec{\epsilon} \cdot \vec{j})$ can be obtained experimentally by measuring the recoil-free resonant cross section of an appropriate magnetic hyperfine component in a magnetically oriented absorber for linearly polarized gamma rays. Let the propagation vector $\overrightarrow{\mathrm{k}}$ and linear polarization vector $\vec{\epsilon}$ of the incident resonant radiation be directed along the $z$ and $x$ axes of a Cartesian coordinate system, respectively. Let the direction of the magnetic field at the nuclei of the magnetized absorber be specified by the azimuthal and polar angles $\alpha$ and $\beta$ respectively. The cross section for a $\Delta m= \pm 1$ hyperfine component in the absorber is then 